\title{
Effect of Risk Management in Diabetic Retinopathy
}

\author{
Junnan Lin ${ }^{1}$, Ye Li ${ }^{1, *}$, Lishi Luo ${ }^{2}$ \\ ${ }^{1}$ Department of Ophthalmology and Stomatology, The First Affiliated Hospital of Jinan University, Guangzhou, China \\ ${ }^{2}$ Ophthalmology Department, The First Affiliated Hospital of Jinan University, Guangzhou, China
}

Email address:

1075184194@qq.com (Junnan Lin), li58828@163.com (Ye Li), 296354125@qq.com (Lishi Luo)

${ }^{*}$ Corresponding author

\section{To cite this article:}

Junnan Lin, Ye Li, Lishi Luo. Effect of Risk Management in Diabetic Retinopathy. International Journal of Diabetes and Endocrinology. Vol. 5, No. 1, 2020, pp. 6-8. doi: 10.11648/j.ijde.20200501.12

Received: December 28, 2019; Accepted: January 6, 2020; Published: January 21, 2020

\begin{abstract}
Objective: To evaluate the effect of risk management of nursing in diabetic retinopathy patient. Methods: 98 patients diagnosed as diabetic retinopathy from January 2018 to January 2019, they were randomly assigned to control group and intervention group, we use the different nursing measure to both of groups. Our researchers collected the data of negative patient reaction and result of serum inflammatory cytokines in before treatment and after treatment. Additionally, we collected the information associated with quality of life on participants by the short form-36 (SF-36). Result: The results of intervention group were better than the results of control group in negative patient reaction [n (\%), $1(2.0 \%)$ vs $6(12.2 \%), p<0.005]$. In addition, the serum inflammatory cytokines and quality of life were improved after treatment, the improvements of intervention group were better than outcome of control group, most result of them had statistical significance $(p<0.005)$. Conclusion: The risk management of nursing improved the outcome of diabetic retinopathy treatment. Although improvement of negative patient reaction was non-significant in result, other aspect of patient had significantly improvement which were serum inflammatory cytokines and quality of life.
\end{abstract}

Keywords: Diabetic Retinopathy, Risk Management, Nursing

\section{Introduction}

Diabetes is a group of metabolic diseases characterized by hyperglycemia resulting from defects in insulin secretion, insulin action, or both [1]. Diabetes had a major impact on the lives, well-being of individuals, families, and societies worldwide, it is a serious and long-term condition [2]. In 2017, global health expenditure on diabetes was estimated to be USD 727 billion [3, 4]. Additionally, diabetic retinopathy is the major eye complication of diabetic patients, it also is the major cause of blindness in the working population $[5,6]$. In diabetic retinopathy, it had two categories that included nonproliferative (NPDR) and proliferative (PDR) [7]. The characteric of PDR was the growth of new blood vessels on the optic disc or elsewhere in the retina, which was occurs with severe retinal ischemia [8]. Following to an aging population and increased prevalence of diabetes, cases of diabetic retinopathy were increase from 4 million to 7.7 million between 2000 and 2010 [9, 10].

The laser therapies were the traditional standard of care for diabetic retinopathy. However, treatment paradigm was shifted as the identification of vascular endothelial growth factor as a key mediator in diabetic retinopathy pathogenesis $[11,12]$. The risk management was important for improving patients in diabetic retinopathy and serum inflammatory cytokines. This paper aim to evaluate the influence of risk management in diabetic retinopathy and serum inflammatory cytokines.

\section{Methods}

\subsection{Participants Enrollment}

The patients $(n=98)$ who were diagnosed as diabetic retinopathy were investigated to join our study. Their diagnosis of time were between January 2018 and January 2019 , they were randomly assigned to a intervention group (n $=49)$ and a control group $(\mathrm{n}=49)$. Additionally, the patients complete the check, the questionnaire and the interview, such as negative patient reaction, serum inflammatory cytokines of before and after nursing and quality of life (QoL) by the Short 
Form-36 (SF-36) [13].

Their inclusion criteria were: (1) was diagnosed as diabetic retinopathy; (2) their retinopathy is caused by diabetes; (3) had no eye surgery; (4) was mentally normal and voluntarily participated in the study. Their withdraw criteria were: (1) the age was higher than 60 years; (2) had abnormal intraocular pressure; (3) had glaucoma, severe conjunctivitis and other eye diseases; (4) had heart diseases, lung diseases, kidney diseases and other major diseases. In addition, written informed consent was obtained from all participants, and this study was approved by the local ethics committee.

\subsection{Survey Methods}

In the control group, doctors and nurses provide routine nursing measures. In the intervention group, we used Implement risk management on the basis of routine care. We collected the information associated with negative patient reaction, serum inflammatory cytokines and QoL in before and after treatment. The negative patient reaction included nausea and vomiting, gastrointestinal reaction and hypoglycemia, our researchers would recording the frequency in the nursing process. In addition, the serum inflammatory cytokines included 3 indexes, such as Tumor Necrosis Factor- $\alpha$ (TNF- $\alpha$ ), Interleukin-6 (IL-6) and C-reactionprotein (CRP). We collected the score of QoL by SF-36, its domains included emotion function, social function, role function and cognitive function.

\subsection{Statistical Analysis}

We performed the statistical analysis by SPSS24.0. The mean standard deviation (measurement data) for statistical description. Additionally, we used $\mathrm{P}$ value, $\mathrm{t}$-test and chi-square test to compare and analyze the result of collection.

\section{Result}

Our researchers collected the information associated with negative patient reaction of patients after treatment (Table 1). Following to Table 1, the cases of negative patient reaction of intervention group was less than control group [n (\%), 1 $(2.0 \%)$ vs $6(1.2 \%)]$, it had statistical significance $(\mathrm{p}<0.005)$.

Table 1. Negative Patient Reaction.

\begin{tabular}{|c|c|c|c|c|c|}
\hline Project & Cases & Nausea and Vomiting & Gastrointestinal Reaction & Hypoglycemia & Total \\
\hline Intervention Group & $49(50 \%)$ & 0 & 1 & 0 & $1(2.0 \%)$ \\
\hline Control Group & $49(50 \%)$ & 2 & 2 & 2 & $6(12.2 \%)$ \\
\hline $\mathrm{X}^{2}$ & - & - & - & - & 4.24 \\
\hline P Value & - & - & - & - & $<0.005$ \\
\hline
\end{tabular}

In serum inflammatory cytokines index, the intervention group had better improvement than the control group, most of result had statistical significance (Table 2). In TNF- $\alpha$, the patients of intervention group had significant improvement, which was from $58.6 \pm 39.2 \mathrm{ng} / \mathrm{L}$ to $29.5 \pm 8.8 \mathrm{ng} / \mathrm{L}$.

Table 2. Serum Inflammatory Cytokines Index.

\begin{tabular}{|c|c|c|c|c|c|c|}
\hline \multirow{2}{*}{ Project } & \multicolumn{2}{|c|}{ TNF- $\alpha$ (ng/L) } & \multicolumn{2}{|c|}{ IL-6 (ng/L) } & \multicolumn{2}{|c|}{ CRP (ng/L) } \\
\hline & Before & After & Before & After & Before & After \\
\hline Intervention Group (Mean \pm SD) & $58.6 \pm 39.2$ & $29.5 \pm 8.8$ & $18.3 \pm 3.7$ & $9.8 \pm 3.5$ & $12.5 \pm 2.6$ & $6.3 \pm 1.7$ \\
\hline Control Group (Mean \pm SD) & $59.3 \pm 8.5$ & $43.1 \pm 7.6$ & $18.6 \pm 3.8$ & $15.4 \pm 3.3$ & $12.5 \pm 2.8$ & $9.5 \pm 2.2$ \\
\hline T-test & 1.72 & 15.93 & 1.53 & 14.83 & 1. 75 & 14.53 \\
\hline $\mathrm{P}$ Value & $<0.005$ & $<0.005$ & 0.012 & $<0.005$ & 0.041 & $<0.005$ \\
\hline
\end{tabular}

Before $=$ We collected the data before the treatment.

After $=$ We collected the data after the treatment.

TNF- $\alpha=$ Tumor Necrosis Factor- $\alpha$.

IL-6 = Interleukin-6.

$\mathrm{CRP}=\mathrm{C}$-reactionprotein.

In score of Qol, it included 4 domains for the patients in this study. The nursing measure associated with risk management significantly improved patients' quality of life in intervention group. In control group, the quality of patient had slight improvement after treatment. Additionally, most of result in 4 domains had statistical significance.

Table 3. Quality of Life (SF-36).

\begin{tabular}{|c|c|c|c|c|c|c|c|c|}
\hline \multirow{2}{*}{ Project } & \multicolumn{2}{|c|}{ Emotion Function } & \multicolumn{2}{|c|}{ Social Function } & \multicolumn{2}{|c|}{ Role Function } & \multicolumn{2}{|c|}{ Cognitive Function } \\
\hline & Before & After & Before & After & Before & After & Before & After \\
\hline Intervention Group (Mean \pm SD) & $62.4 \pm 2.8$ & $85.1 \pm 12.5$ & $62.6 \pm 5.5$ & $88.2 \pm 7.8$ & $62.4 \pm 6.6$ & $82.3 \pm 8.7$ & $63.4 \pm 5.2$ & $81.7 \pm 6.5$ \\
\hline Control Group (Mean \pm SD) & $61.6 \pm 6.2$ & $66.7 \pm 9.4$ & $62.6 \pm 5.4$ & $71.1 \pm 6.3$ & $61.7 \pm 6.4$ & $68.6 \pm 7.5$ & $62.7 \pm 5.1$ & $69.7 \pm 5.4$ \\
\hline T-test & 1.5247 & 14.753 & 1.635 & 14.756 & 1.845 & 15.826 & 1.473 & 14.826 \\
\hline $\mathrm{P}$ Value & 0.031 & $<0.005$ & $<0.005$ & $<0.005$ & 0.033 & $<0.005$ & $<0.005$ & $<0.005$ \\
\hline
\end{tabular}

Before $=$ We collected the data before the treatment.

After $=$ We collected the data after the treatment.

SF-36 $=$ The Short Form-36. 


\section{Discussion}

Diabetic retinopathy is one of the most prominent pathologic vascular complications of diabetes, it is the most common cause of blindness in the working-age popular [14]. In China, common risk factors for the development of DR include duration of diabetes, poor glycemic control, elevated blood pressure, presence of diabetic nephropathy and dyslipidemia [15]. As some report, the risk factors include increased duration of diabetes mellitus, as well as severity of hypertension and hyperglycemia [16-18].

According to the result of collection and analyse, the risk management of nursing slightly improved negative patient reaction, the cases of negative patient reaction of intervention group were less than the cases of control group in nausea and vomiting, gastrointestinal reaction and hypoglycemia. But the influence of risk management of nursing had not significant effect. In serum inflammatory cytokines index, the patients had significant improvement after treatment in intervention group, the most result of serum inflammatory cytokines had statistical significance. So risk management of nursing can improve serum inflammatory cytokines of patient in nursing process. Additionally, the risk management of nursing also can improve the QoL of patient. Especially in the social function domain, its influence was better than another domain. The result of intervention group had better improvement than the result of control group. Besides, the social function domain and cognitive function domain had statistical significance.

\section{Conclusion}

In summary, the risk management of nursing was good for improving outcome of diabetic retinopathy treatment. It can improve three aspects of patient, such as negative patient reaction, serum inflammatory cytokines and QoL. However, this result only assess the effect what the patients were treated in The First Affiliated Hospital of Jinan University of China and only assess the influence of our risk management of nursing as the part of nursing measure was different for other study.

\section{References}

[1] American Diabetes Association. Standards of medical care in diabetes 2018. January, vol. 41; 2018. Suppl. 1.

[2] Chatterjee S, Khunti K, Davies MJ. Type 2 diabetes. Lancet Lond Engl. 2017, 389, 10085, 2239-2251.
[3] International Diabetes Federation. IDF Diabetes Atlas, 8th ed. Brussels. Belgium: International Diabetes Federation. 2017.

[4] wanga FS, Njelekela MA, Diamond MB, et al. Urban and rural prevalence of diabetes and pre-diabetes and risk factors associated with diabetes in Tanzania and Uganda. Glob Health Action. 2016, 23 (9), 31440.

[5] Agarwal P, Jindal A, Sainiv K, Jindal S. Advances in diabetic retinopathy $[\mathrm{J}]$. Indian J Endoainol Metab, 2014, 18 (6), $772-777$.

[6] Sayin N, Kara N, Pekel G. Ocular complications of diabetes mellitus. World J Diabetes. 2015, 6 (1), 92-108.

[7] Mohamed Q, Gillies MC, Wong TY. Management of diabetic retinopathy: a systematic review. JAMA, J Am Med Assoc. 2007, 298 (8), 902-916.

[8] Singh R, Ramasamy K, Abraham C, et al. Diabetic retinopathy: an update. Indian J Ophthalmol. 2008, 56 (3), 178-188.

[9] National Eye Institute. Diabetic retinopathy. https://nei.nih.gov/eyedata/diabetic. Accessed 15 July 2017.

[10] Takahashi H, Tampo H, Arai Y, Inoue Y, Kawashima H. Applying artificial intelligence to disease staging: deep learning for improved staging of diabetic retinopathy. PLoS One. 2017, 12, 87-90.

[11] Solomon SD, Chew E, Duh EJ, Sobrin L, Sun JK, VanderBeek BL, et al. Diabetic retinopathy: a position statement by the American Diabetes Association. Diabetes Care. 2017, 40, 412-418.

[12] Ip MS, Domalpally A, Sun JK, Ehrlich JS. Long-term effects of therapy with ranibizumab on diabetic retinopathy severity and baseline risk factors for worsening retinopathy. Ophthalmology. 2015, 122, 367-74.

[13] Ware JE. SF-36 health survey update. Spine. 2000, 25, 3130 3139 .

[14] Jorge R, Costa RA, Comt DC, et al. Intravitreal bevacizumab (Avastin) for persistent new vessels in diabetic retinopathy (IBEPE Study). Retina. 2006, 26, 1006-1013.

[15] Malaguarnera G, Gagliano C, Bucolo C, et al. Lipoprotein (a) in cardiovascular diseases. BioMed Res Int. 2013, 1-5.

[16] Tan GS, Gan A, Sabanayagam C, et al. Ethnic differences in the prevalence and risk factors of diabetic retinopathy: the singapore epidemiology of eye diseases study. Ophthalmology. 2018, 125 (4), 529-536.

[17] Tomita M, Kabeya Y, Okisugi M, Katsuki T, Oikawa Y, Atsumi $\mathrm{Y}$, et al. Diabetic microangiopathy is an independent predictor of incident diabetic foot ulcer. J Diabet Res. 2016, 5938540. 